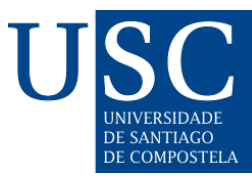

\title{
Sostenibilidad Corporativa y Objetivos de Desarrollo Sostenible: innovación para luchar contra la corrupción
} Corporate Sustainability and Sustainable Development Goals: innovation to fight against corruption

\author{
Joaquín Fernández Mateo ${ }^{1, a, * \mathbb{D}}$, Julia Jambrina Rodríguez ${ }^{2, b, * *}$ (D) \\ ${ }^{1}$ Universidad Rey Juan Carlos, España \\ ${ }^{2}$ Universidad de Extremadura, España \\ a joaquin.fernandez@urjc.es b jjambrina@unex.es
}

Recibido: 01/02/2021; Aceptado: 24/07/2021

\section{Resumen}

Desde la definición de Bowen, la responsabilidad social moderna ha sufrido muchas transformaciones. Paralelamente, el concepto de sostenibilidad ha superado sus límites medioambientales. En consecuencia, la sostenibilidad corporativa es un concepto que integra los aspectos financieros, sociales y medioambientales, sin dejar de reconocer la importancia del gobierno corporativo como clave de todo el proceso empresarial. De hecho, algunos estudios empíricos han demostrado que el gobierno corporativo es clave para lograr la sostenibilidad corporativa. Esto nos lleva a estudiar la responsabilidad de la alta dirección en la implementación de las políticas de responsabilidad social. Dentro de los Objetivos de Desarrollo Sostenible (ODS), la innovación tecnológica (ODS 9) puede disminuir los riesgos de corrupción, aumentar la transparencia y fomentar la rendición de cuentas (ODS 16). Los datos de distintas organizaciones demuestran que los países más innovadores se sitúan en los mejores puestos en materia de corrupción. En este sentido, el gobierno corporativo puede alcanzar la sostenibilidad corporativa a través de la innovación que proporcionan las nuevas tecnologías. Automatización, análisis de grandes datos en búsqueda de patrones, correlaciones o anomalías, redes sociales y canales de información y comunicación pueden disminuir los riesgos asociados a la corrupción, aumentando la accountability. Aumentar la inversión en innovación y posibilitar sinergias mediante la colaboración público-privada — posibilidades planteadas en los ODS 9 y ODS 17-, pueden permitir alcanzar las metas específicas del ODS 16: disminuir la corrupción, aumentar la transparencia y mejorar la rendición de cuentas.

Palabras clave: sostenibilidad corporativa; gobierno corporativo; ODS; innovación; tecnología.

\section{Abstract}

Modern social responsibility has undergone lots of transformations, since Bowen's defined it. Simultaneously, the concept of sustainability is no longer just an environmental concept. Consequently, corporate sustainability is a notion that joins financial, social and environmental aspects, while still recognizing the importance of corporate governance as the key to the entire business development. Some empirical studies have actually shown that corporate governance is essential to achieving corporate 
sustainability. This leads us to study the responsibility of senior management in the implementation of social responsibility policies. Within the Sustainable Development Goals (SDGs), technological innovation (SDG 9) may reduce the risks of corruption, increase commitment to transparency, and encourage a work culture of accountability (SDG 16). Reports and statistics from different organizations show that the more innovative countries are the less corrupt. In this context, corporate governance can achieve corporate sustainability through the innovation provided by new technologies. Automation process can be used for better decision making, preventing fraudulent activities as well as analysis of big data that would provide search of patterns, correlations or anomalies, social networks and information and communication channels. Besides, it can reduce the risks associated with corruption, increasing accountability. Increasing investment in innovation and enabling synergies through public-private collaboration — possibilities outlined in SDGs 9 and SDG 17 - can make it possible to achieve the specific goals of SDG 16: decrease corruption, increase transparency and improve accountability in organizations.

Keywords: corporate sustainability; governance; SDG; innovation; technology.

\section{Sumario}

1. Introducción e hipótesis de investigación

2. Responsabilidad social y sostenibilidad corporativa

3. Sostenibilidad corporativa y gobierno corporativo

4. Accountability

5. Innovación contra la corrupción

6. Conclusión

\section{Summary}

1. Introduction and research hypothesis

2. Social responsibility and corporate sustainability

3. Corporate sustainability and corporate governance

4. Accountability

5. Innovation against corruption

6. Conclusion

\section{INTRODUCCIÓN E HIPÓTESIS DE INVESTIGACIÓN}

La Agenda 2030 de las Naciones Unidas — con 17 Objetivos de Desarrollo Sostenible, 169 metas y 232 indicadores - cumple un papel esencial para identificar y erradicar los problemas de pobreza y sostenibilidad de la comunidad internacional. La Agenda contempla problemas relacionados con diferentes cuestiones como el desarrollo humano, la sostenibilidad medioambiental, el desarrollo económico y la prosperidad, la paz y las alianzas, así como los medios para su seguimiento, revisión y, en definitiva, su posible solución. Las organizaciones públicas y privadas están utilizando la Agenda 2030 como una hoja de ruta para comprometerse con la sostenibilidad. En este trabajo estudiaremos las posibles soluciones para alcanzar el ODS 16, que busca Promover sociedades más pacíficas e inclusivas con instituciones efectivas y responsables, y plantea dos metas específicas: Reducir considerablemente la corrupción y el soborno en todas sus formas (16.5) y Crear a todos los niveles instituciones eficaces y transparentes que rindan cuentas (16.6).

La corrupción tiene consecuencias para el resto de ODS pues "is one of the world's most pervasive problems, affecting international trade, environmental protection efforts, human rights, national 
security, access to health care and justice services, economic development, and the legitimacy of governments around the world" (Feathers, 2014: 287). Además, se ha demostrado, que la corrupción tiene también efectos negativos sobre los índices de pobreza (ODS 1), el índice de desarrollo humano (ODS 1-5), la seguridad alimentaria (ODS 2), las tasas de mortalidad (ODS 3), las tasas de abandono escolar (ODS 4), el suministro de agua (ODS 6), la conservación de la naturaleza (ODS 15) y la confianza en los gobiernos (ODS 16) (Trapnell, Jenkins \& Chene, 2017: 36).

Las empresas, como agentes sociales, pueden jugar un papel importante en la consecución de los ODS. Para ello, no solo deben rendir cuentas ante propietarios y accionistas, sino ante el "mapa topográfico de sus stakeholders", avanzando hacia una gestión "no sólo eficiente, sino también socialmente responsable y éticamente correcta. Es decir: una gestión a la altura de las demandas del momento en lo económico, pero también en lo humano y lo moral" (FernándezFernández \& Bajo Sanjuán, 2012: 139). En este escenario económico —no exento de una crisis de confianza - juega un papel importante la tecnología; la innovación responsable caracterizaría a cualquier empresa "que quisiera asentar su actividad, verdaderamente, a la altura de los tiempos" (Benavides \& Fernández, 2020: 18). Pero esa responsabilidad social de la empresa tiene una larga historia que hay que detallar. Desde la definición de Bowen (1953), la responsabilidad social moderna ha recibido diferentes descripciones y significados (Carroll, 1979; Hoffman, 1986; Dahlsrud, 2006; 0'Mara-Shimek, Guillén \& Bañón, 2015; Díaz-De la Cruz \& Fernández-Fernández, 2016; Fernández-Mateo \& Franco-Barrera, 2020). Paralelamente, el concepto de sostenibilidad (Brundtland, 1987) ha trascendido sus límites ambientales. La sostenibilidad corporativa es un concepto que integra, además de los aspectos financieros, elementos sociales y ambientales (Elkington, 1997), alcanzando a los consejos de administración (Elkington, 2006):

When transposing this idea to the business level, corporate sustainability can accordingly be defined as meeting the needs of a firm's direct and indirect stakeholders (such as shareholders, employees, clients, pressure groups, communities, etc.), without compromising its ability to meet the needs of future stakeholders as well. Towards this goal, firms have to maintain and grow their economic, social and environmental capital base while actively contributing to sustainability in the political domain (Dyllick \& Hockerts, 2002: 131-132)

Siguiendo la literatura científica de la materia, algunos estudios han demostrado que el buen gobierno corporativo es clave para alcanzar la responsabilidad social (Harjoto \& Jo, 2011; Jo \& Harjoto, 2012). Es decir, la clave de la sostenibilidad corporativa está en el gobierno corporativo, governance en su acepción inglesa. Esto nos lleva a estudiar la responsabilidad de la alta dirección y de los consejos de administración en el establecimiento de una política coherente de responsabilidad social corporativa o, siguiendo su evolución histórica, de sostenibilidad corporativa.

El nuevo gobierno corporativo tiene la responsabilidad de elaborar las políticas que permitan alcanzar la sostenibilidad corporativa. Para cumplir con algunas dimensiones esenciales del gobierno corporativo, la innovación y las nuevas tecnologías son elementos que pueden aumentar la transparencia y la rendición de cuentas (Bovens, Schillemans \& Goodin, 2014). En este punto, el ODS 16 conecta con el ODS 9. El ODS 9 plantea que la innovación y el progreso tecnológico son claves para descubrir soluciones duraderas para los desafíos económicos y medioambientales, como el aumento de la eficiencia energética y de recursos. Además, la meta 9.5 propone Aumentar la investigación científica y mejorar la capacidad tecnológica de los sectores industriales de todos los países, en particular los países en desarrollo, entre otras cosas fomentando la innovación. Y esta innovación se relaciona con el ODS 16 porque, a través de la innovación, las nuevas herramientas 
tecnológicas pueden reducir la corrupción, creando organizaciones más transparentes que rindan cuentas.

A partir de estos argumentos podemos formular las siguientes hipótesis:

H1: La sostenibilidad corporativa y su dimensión clave, el governance, es condición necesaria, pero no suficiente, para alcanzar organizaciones más sostenibles. La reducción de la corrupción, el aumento de la transparencia y la rendición de cuentas dependen del gobierno corporativo de las organizaciones.

H2: El buen gobierno corporativo, gracias a la innovación tecnológica, puede alcanzar los objetivos corporativos, es decir, la sostenibilidad corporativa. La innovación tecnológica es la clave del buen governance, generando organizaciones más transparentes y sostenibles en el largo plazo.

A continuación, procederemos a definir los elementos fundamentales del trabajo: cómo el concepto sostenibilidad amplía su significado para, a través del concepto de sostenibilidad corporativa, servir de herramienta para lograr mejores empresas. Sobre este trabajo conceptual se argumenta que la clave de la sostenibilidad corporativa reside en un buen governance (H1), donde la noción de accountability es fundamental (ODS 16). Una vez establecidos los fundamentos teóricos del nuevo gobierno corporativo se añade la hipótesis de la innovación (H2; ODS 9) como palanca de cambio que permite alcanzar las metas planteadas en el ODS 16.

\section{RESPONSABILIDAD SOCIAL Y SOSTENIBILIDAD CORPORATIVA}

La Comisión Brundtland elaboró un concepto de desarrollo sostenible que señalaba la necesidad de satisfacer "the needs of the present without compromising the ability of future generations to meet their own needs" (Brundtland, 1987: 8). Desde entonces, el concepto de sostenibilidad ha trascendido las ciencias ambientales, para que las generaciones presentes alcancen "a high degree of economic security and can realize democracy and popular participation in control of their communities, while maintaining the integrity of the ecological systems upon which all life and all production depends" (Viederman, 1994: 5). Esta ampliación del concepto ha permeado la teoría de la empresa, que centrará su atención en el impacto medioambiental de las actividades empresariales al mismo tiempo que persigue sus metas financieras. Pero, precisamente por el carácter integral del concepto —algo más que la reducción de los impactos ambientales — algunos trabajos lo han aproximado al campo de la ética, en concreto, a la práctica de las virtudes:

Sustainability refers to a moral way of acting, and ideally habitual, in which the person or group intends to avoid deleterious effects on the environmental, social, and economic domains, and which is consistent with a harmonious relationship with those domains that is conducive to a flourishing life. (Bañon Gomis, Guillén Parra, Hoffman \& McNulty, 2011: 176)

Pero éste no es sino el final de un lento proceso de cuestionamiento académico y evolución conceptual que es necesario describir. Para ello, es necesario remontarse a los orígenes de la responsabilidad social. El origen de la responsabilidad social moderna es atribuido al economista norteamericano Howard Bowen, que utilizó el término en su trabajo Social Responsibility of a Businessman. Para Bowen (1953: 6), "the responsibility of the businessman refers to the obligations to pursue those policies, to make those decisions, or to follow those lines of action which are desirable in terms of the objectives and values of our society”. Años más tarde, en su obra de 1962, Capitalism and Freedom, Milton Friedman argumentó que: 
There is one and only one social responsibility of business: to use its resources and engage in activities designed to increase its profits so long as it stays within the rules of the game, which is to say, engages in open and free competition, without deception or fraud (Friedman, 1962: 112).

Frente al modelo clásico de Friedman, en un trabajo muy influyente, Edward Freeman (1984) describió a los stakeholders de una empresa como aquellos que pueden afectar a la empresa o verse afectados por ella. Este planteamiento, mucho más pluralista, llevó a reconsiderar la identidad de las organizaciones; éstas se formarían y reformarían en función de las relaciones con sus diversos stakeholders:

The broadest definition of 'stakeholder' brings in anyone who affects or is affected by a company's operations. The key new perception is that companies need to expand the range of interests considered in any new development from customers, shareholders, management, and employees to such people as suppliers, local communities, and pressure groups (Elkington, 1997: 397)

De esta forma, el término stakeholders señaló aquellos elementos esenciales de la planificación estratégica de las compañías. La adecuada gestión del ecosistema empresarial será la clave del éxito de la organización, siendo la teoría de los stakeholders el paradigma dominante en RSC (McWilliams \& Siegel, 2001). Para Elkington (1994: 97), la preocupación por cuestiones ambientales dio lugar a un conjunto creciente de "involuntary, mandatory, and voluntary forms of corporate environmental disclosure". A partir de entonces, los aspectos sociales y ambientales se convierten en imperativos que, junto a los resultados financieros, definen el esquema tridimensional e interdependiente de los negocios sostenibles. Para Elkington (1997: 397), el desarrollo sostenible "involves the simultaneous pursuit of economic prosperity, environmental quality, and social equity. Companies aiming for sustainability need to perform not against a single, financial bottom line but against the triple bottom line".

Un ejemplo de la materialización de los esfuerzos en responsabilidad social —en su evolución hacia la sostenibilidad corporativa - son los informes de sostenibilidad. El Global Reporting Initiative (GRI) es la institución independiente constituida en 1997 cuyo principal objetivo es armonizar y estandarizar la elaboración de memorias de sostenibilidad para aquellas empresas que deseen medir y reportar su desempeño económico, social y medioambiental (Andreu, Fernández \& Fernández, 2019). Sin embargo, no han sido pocas las críticas que han visto cómo los estándares GRI se han desviado de sus objetivos principales - convirtiéndose en una herramienta para los objetivos particulares de las compañías-. Este es un ejemplo de cómo el concepto de sostenibilidad debe ser analizado con precisión para combatir su posible ambigüedad; un término potente que, sin embargo, puede vaciarse de contenido fácilmente (Benavides \& Fernández, 2020). Esto es lo que ocurre cuando no sabemos quiénes son los verdaderos beneficiarios de ella:

The process of the development of the GRI guidelines has meant an opportunity for the different lobbies to further their own (environmental) agendas by appropriating these concepts. More and more companies are adopting the GRI methodology to prepare their sustainability reports but, at the same time, the level of compromise with sustainable development assumptions is low. As a consequence, the guidelines developed by the GRI are used as a new tool for legitimising management decisions and actions. The acceptance of sustainability reports without restrictive conditions such as a clear definition of entity boundaries, the development/ requirement of integrated indicators or the attachment of an independent verification statement leads to a relaxation of the basic aim, that is, sustainability (Moneva, Archel \& Correa, 2006: 134)

A pesar de la dificultad de materializar un concepto tan complejo y un proceso de transformación sistémico y multilateral, este conjunto de preocupaciones, ideas, iniciativas, recomendaciones y 
prácticas cristalizaron en el Pacto Mundial (Global Compact), de 31 de enero de 1999. El Pacto Mundial, emanado de la ONU, no nació ni como un organismo para la formulación de normativa internacional ni como un código de conducta internacional con fuerza jurídica obligatoria, pero dará impulso a toda una serie de iniciativas multilaterales en materia de derechos humanos, trabajo, medio ambiente y lucha contra la corrupción. Ejemplos de ello son la aparición de los índices de sostenibilidad, herramientas que buscan medir el desempeño sostenible, entendido como la capacidad de integrar la economía, el medio ambiente y los factores sociales en la gestión de las compañías:

Los índices de sostenibilidad relacionan el éxito económico de un producto con el desempeño sostenible de la compañía; a largo plazo, las compañías generan unos resultados más predecibles, lo que significa menos sorpresas y más confianza. [...] Mediante estos índices se ponen en relación las variables económicas con la variable tridimensional de la sostenibilidad (social, ambiental y buen gobierno). Los inversores pueden excluir de su cartera aquellas compañías que no respeten los derechos humanos, el medio ambiente o generar sus rendimientos económicos a partir de actividades éticamente cuestionables (Andreu, Fernández \& Fernández, 2018: 24)

El Pacto Mundial estableció, en su principio 10, que las empresas e instituciones deberán trabajar contra la corrupción en todas sus formas, incluidos extorsión y soborno. Sin embargo, desde entonces, la materialización de este principio no se ha producido. Tras la crisis financiera y la consiguiente pérdida de confianza en las organizaciones económicas y empresariales, el universo empresarial e institucional adolece de un proceso de falta de credibilidad (Benavides \& Fernández, 2020). Pese a todas las iniciativas encaminadas a luchar contra la corrupción, esta lacra afecta a todo tipo de instituciones, organizaciones y países.

Las sucesivas cumbres, acuerdos e instrumentos multilaterales dieron lugar a la aparición de los Objetivos del Milenio, en el año 2000, y los Objetivos de Desarrollo Sostenible, en 2015. La diferencia entre ambas iniciativas reside en que mientras "los Objetivos del Milenio tenían un enfoque social limitado a los países en desarrollo, los Objetivos de Desarrollo Sostenible tienen un enfoque holístico, interconectado o sistémico, que afecta a todos los países" (Andreu, Fernández y Fernández, 2019). La Agenda 2030 supone un nuevo impulso a la lucha por la transparencia y contra la corrupción. Dentro de estos 17 ODS, el ODS 16 aboga por construir sociedades más pacíficas e inclusivas, con instituciones efectivas y responsables. Para lograrlo es necesario que se establezcan reglamentaciones más eficientes y transparentes, y presupuestos gubernamentales integrales y realistas. No en vano, la corrupción, el soborno, el robo y la evasión impositiva cuestan alrededor de 1,26 billones de dólares para los países en desarrollo por año (Naciones Unidas, s.f.). Estas cantidades podrían ser utilizadas para luchar contra la pobreza y reducir la desigualdad. Por esto, el ODS 16 plantea como metas Reducir considerablemente la corrupción y el soborno en todas sus formas (16.5) y Crear a todos los niveles instituciones eficaces y transparentes que rindan cuentas (16.6).

\section{SOSTENIBILIDAD CORPORATIVA Y GOBIERNO CORPORATIVO}

Diferentes estudios (Waddock \& Graves, 1997; Stanwick \& Stanwick, 1998; Choi, Kwak \& Choe, 2010; Inoue \& Lee 2011; Eccles, Ioannou \& Serafeim, 2014; Khan, Serafeim \& Yoon, 2016) han demostrado que las empresas que adoptan políticas corporativas relacionadas con el medio ambiente, los empleados, la comunidad y la cadena de valor superan a sus competidores en 
términos financieros en el largo plazo. Las empresas de baja sostenibilidad siguen el modelo tradicional de maximización del beneficio empresarial, en el que las cuestiones sociales y medioambientales se consideran predominantemente como externalidades que acaban siendo responsabilidad de los gobiernos. Las empresas de alta sostenibilidad, en cambio, no solo prestan atención a las externalidades, sino que de hecho se caracterizan por tener mecanismos de gobernanza que involucran directamente al consejo de administración en temas de sostenibilidad, mejorando la reputación y reduciendo los riesgos. En consecuencia, "firms should integrate social and environmental initiatives in their core business strategies to gain a competitive advantage that will enable them to improve their corporate financial performance" (Marti, Rovira-Val \& Drescher, 2015: 316).

Involucrar al consejo de administración en temas de sostenibilidad se propone como la clave de la sostenibilidad corporativa, es decir, es un problema de governance. El gobierno corporativo es algo más amplio que la gestión empresarial. Implica una administración justa y transparente de las organizaciones. En su definición clásica, el gobierno corporativo establece las reglas básicas que determinan "who has what control rights under what circumstances, who receives what share of the wealth created, and who bears what associated risks. Governance systems thus help determine how decisions are made about spending resources on building organizational capabilities" (Blair, 1996: 17). Más recientemente, para la OCDE (2016), el gobierno corporativo contribuirá a un crecimiento más sólido al facilitar la creación de un ambiente de confianza, transparencia y rendición de cuentas, necesario para favorecer las inversiones a largo plazo, la estabilidad financiera y la integridad en los negocios.

Las metas clásicas del consejo de administración son gestionar de forma adecuada la compañía, obtener legitimidad social y lograr los recursos necesarios para su supervivencia (FernándezFernández, 1999). Para alcanzar dichos logros, la trasparencia es clave. El buen gobierno está asociado a una mayor transparencia y una mayor claridad de la información financiera. El mal gobierno corporativo se relaciona con el engaño en el estado financiero, poniendo en peligro la sostenibilidad corporativa. Para Beltratti (2005: 384) "corporate governance and corporate social responsibility are therefore complementary" and "firms with a good corporate governance are also more respected and valuable. Therefore a good corporate governance protects the stakeholders which contribute to the life of the firm" (2005: 385).

Un buen gobierno corporativo supone la estructuración sistemática de las operaciones de una empresa para lograr sus objetivos estratégicos. Estos implican la satisfacción de los accionistas, acreedores, empleados, clientes y proveedores, conjuntamente con el cumplimiento de los requisitos legales, reglamentarios y los compromisos sociales. En consecuencia, el gobierno corporativo adquiere un significado amplio que implica el respeto por el entorno donde la organización desarrolla su actividad, es decir, tanto criterios de sostenibilidad ambiental como el respeto de las comunidades locales. El gobierno corporativo es clave para lograr la sostenibilidad corporativa y, en consecuencia, para conseguir las metas planteadas en el ODS 16. Si inicialmente el gobierno corporativo "deals with the ways in which suppliers of finance to corporations assure themselves of getting a return on their investment" (Shleifer \& Vishny, 1997: 737), posteriormente la sostenibilidad corporativa en su triple dimensión entrará en las preocupaciones de los consejos de administración:

Corporate governance is fundamentally about such questions as what business is for - and in whose interests companies should be run, and how. Wider issues such as business ethics through entire value chains, human rights, bribery and corruption, and climate change are among the great issues of our time that increasingly cross- 
cut the rarified worlds of corporate boardrooms. As a result, a growing proportion of our own work has seen the spot-welding of corporate governance with such wider societal concerns. As a result, it's timely to review the increasingly complex cross-connects between the rapidly mutating governance agenda and the burgeoning world of corporate responsibility, social entrepreneurship and sustainable development. (Elkington, 2006: 522).

La integración del buen gobierno corporativo —como dimensión clave de la sostenibilidad empresarial— en los consejos de administración garantiza el retorno de la inversión y la vida de la compañía. De hecho, algunos estudios han demostrado que el gobierno corporativo es clave para alcanzar la responsabilidad social o sostenibilidad corporativa (Harjoto, M. A. \& Jo, H., 2011; Jo, H. \& Harjoto, M. A., 2012). Es decir, la clave de la sostenibilidad corporativa está en el gobierno corporativo (Andreu et al., 2020), siendo la dimensión Soborno, Corrupción y Blanqueo de Capitales (ver Tabla 1) una de sus dimensiones más relevantes.

Tabla 1. Elementos de la dimensión Soborno, Corrupción y Blanqueo de Capitales

\begin{tabular}{|l|}
\hline \multicolumn{1}{|c|}{ Soborno, Corrupción y Blanqueo de Capitales } \\
\hline $\begin{array}{l}\text { Exposición de la compañía a riesgo de soborno y corrupción por operaciones en } \\
\text { países de alto riesgo. }\end{array}$ \\
\hline Elaboración de una política para luchar contra el soborno y la corrupción. \\
\hline Elaboración de una política para luchar contra el blanqueo de capitales. \\
\hline $\begin{array}{l}\text { Establecimiento de un programa para luchar contra el soborno, la corrupción y el } \\
\text { blanqueo de capitales mediante la comunicación y la formación de los empleados, } \\
\text { los procesos de seguimiento y reporte al consejo, y los ejercicios de diligencia } \\
\text { debida. }\end{array}$ \\
\hline Controversias o incidencias en temas relacionados con la ética del negocio. \\
\hline $\begin{array}{l}\text { Existencia de canales de denuncia (Whistleblowing) independientes (con firma } \\
\text { externa) y garantía de anonimato. }\end{array}$ \\
\hline Procedimientos para garantizar la independencia de los canales de denuncias. \\
\hline $\begin{array}{l}\text { Transparencia en los pagos a partidos políticos y a las fundaciones dependientes } \\
\text { de éstos, a medios de comunicación y a sindicatos, y conformidad de estos pagos } \\
\text { con la ley. }\end{array}$ \\
\hline $\begin{array}{l}\text { Transparencia en las donaciones benéficas y los patrocinios con instituciones } \\
\text { públicas. }\end{array}$ \\
\hline $\begin{array}{l}\text { Procedimiento de depuración de responsabilidades por malas prácticas en los } \\
\text { procedimientos de contratación y licitación pública. }\end{array}$ \\
\hline
\end{tabular}

Andreu, Fernández \& Fernández, 2020, y elaboración propia.

Por ello, la dimensión gobierno corporativo de la nueva sostenibilidad de empresarial se demuestra fundamental (H1). Si las empresas quieren contribuir a la consecución de los ODS, al mismo tiempo que preocuparse por los aspectos más medioambientales, deben prestar mayor atención a las variables que se deducen de la dimensión soborno, corrupción y blanqueo de capitales. De lo contrario, la contribución a los desafíos planteados por los ODS - recogidos de forma entusiasta por empresas y organizaciones-— será más limitada. No solo no se contribuirá a la realización de las mencionadas metas del ODS 16, sino que afectará a toda una serie de metas asociadas a la lucha contra la pobreza (ODS 1) o la reducción de las desigualdades (ODS 10). 


\section{ACCOUNTABILITY}

Dahl sostiene que en cualquier organización "some individuals have more influence over key decisions than do other" (1958: 465) y esa influencia solo puede analizarse "on the careful examination of a series of concret decisions" (1958: 466). Para Polsby (1963), poder, influencia y control serían sinónimos, y para determinar quién tienen más poder es preciso estudiar quién prevalece en la toma de decisiones. Si la prevalencia en la toma de decisiones determina el poder, esa distinción o desigualdad nos permite determinar la responsabilidad. La formalización de la toma de decisiones permite identificar a los responsables: "an attribution of power is at the same time an attribution of (partial or total) responsibility for certain consequences. The point, in other words, of locating power is to fix responsibility for consequences held to flow from the action, or inaction, of certain specifiable agents" (Lukes, 2005: 58).

Las decisiones estratégicas no formalizadas suelen coincidir con personalidades carismáticas, con una influencia decisiva dentro de una organización. El carácter carismático del directivo — "attributions of charisma increase the power of executives" (Tourigny, Dougan, Washbush, \& Clements, 2003: 1039) — puede desviar la atención del juicio ético de otros miembros de la organización, capaces de valorar aspectos más allá de la mera rentabilidad: "unless a major and visible ethical breach occurs, decisions made and means used by leaders will not be investigated, thereby protecting unethical and illegitimate actions" (2003: 1039). Cuanto más se formalicen las decisiones, más visibilidad habrá en la organización y menos arbitrariedad. Por otro lado, también hay que reconocer el ejercicio del poder por la ausencia de decisiones. Decidir no tratar un determinado tema puede obviar un problema latente o manifiesto (Bachrach \& Baratz, 1979). Decidir no reconocer los problemas es hacerlos invisibles. El silencio moral (Bird, 1996) puede llevar a no denunciar abusos o malas conductas, no disentir de comportamientos moralmente inaceptables, no cuestionar aspectos oscuros de un determinado asunto o no negociar con la suficiente intensidad posiciones que tratan de alcanzar objetivos justos.

Como resultado de este cambio de enfoque — es decir, que la sostenibilidad corporativa se convierte en una dimensión estratégica del alto gobierno de una organización-, la accountability aparece como una de las dimensiones máas relevantes. De la accountability depende la evaluación y seguimiento de la toma de decisiones. Es decir, quién ostenta la autoridad en la organización y, por tanto, quién es responsable último. La atribución de poder es una atribución de responsabilidad. Podemos atribuir poder a aquella persona que, por su posición estratégica en una organización, puede inducir cambios positivos para la organización y sus stakeholders. Si se formaliza la toma de decisiones — quién decide qué- y se identifica a los responsables — frente a un escenario borroso o difuso- es más fácil que el poder no se desdibuje y que pueda haber rendición de cuentas. La accountability permite reunir diferentes conceptos como la transparencia, la responsabilidad, la integridad o la equidad, siendo sinónimo de buen gobierno. Pero, de forma concisa, el concepto de accountability sería:

'the obligation to explain and justify conduct'. This implies a relationship between an actor, the accountor, and a forum, the account-holder or accountee. I will therefore stay close to its etymological and historical roots and define accountability as a specific social relation. Accountability is a relationship between an actor and a forum, in which the actor has an obligation to explain and to justify his or her conduct, the forum can pose questions and pass judgement, and the actor may face consequences. (Bovens, 2007: 450) 
Si una empresa no tiene claro quién toma las decisiones, esa empresa no será responsable. Si no hay rendición de cuentas, no hay justificación para dar explicaciones ante una audiencia, aumentando el riesgo corporativo de una organización: "there needs to be a possibility for the forum to interrogate the actor and to question the adequacy of the information or the legitimacy of the conduct-hence, the close semantic connection between 'accountability' and 'answerability"' (Bovens, 2007: 451). La rendición de cuentas limita los liderazgos carismáticos y permite recompensar a los responsables de la toma de decisiones, vinculando la remuneración de los ejecutivos a los objetivos de sostenibilidad corporativa (Maas \& Rosendaal, 2016; Nigam, Benetti \& Mbarek, 2018). Si una organización es "accountable" es más fácil corregir las malas decisiones identificando a sus responsables y reconociendo los errores. Y de la misma manera, es más fácil premiar las buenas decisiones.

En la mayoría de las grandes empresas, suele haber una clara demarcación entre los responsables de supervisar una política y los responsables de su aplicación cotidiana: "clarity of definition of accountability and responsibility is important in defining roles and responsibilities across organisations generally and within projects that organisations deliver" (McGrath \& Whitty, 2018). La supervisión de la política de la compañía suele ser responsabilidad de la alta dirección o del consejo de administración. En cambio, su aplicación cotidiana suele ser responsabilidad de personas o equipos específicos y abarca tareas como el desarrollo y la aplicación de sistemas y procesos de gestión, el establecimiento de objetivos y metas, la medición y el seguimiento de los resultados y la elaboración de informes. La aparición de un documento de delegación de autoridades es una práctica de buen gobierno (Useem \& Zelleke, 2006).

A lo largo de este trabajo se ha destacado el papel de los consejos de administración en la formulación de las estrategias de las compañías, sugiriendo que los consejos participan de forma significativa en la sostenibilidad de las empresas. De hecho, el Código de Gobierno Corporativo de Sociedades Cotizadas, de la Comisión Nacional del Mercado de Valores (CMNV, 2015), recomienda —Recomendaciones 53 y 54- repartir la supervisión del cumplimiento de la política de RSC entre varias comisiones del consejo de administración, y cuáles deberían ser los contenidos mínimos de la política de RSC. Involucrar al consejo de administración en temas de sostenibilidad es fundamental para alcanzar la sostenibilidad corporativa. Pero lejos de ser una declaración de buenas intenciones, dicha responsabilidad ha cristalizado en el ordenamiento jurídico español. La RSC tiene un lugar en el ordenamiento jurídico español. En particular en la Ley 31/2014 por la que se modifica la Ley de Sociedades de Capital para la mejora del gobierno corporativo, que en su Artículo 529 ter. 1 establece que:

El consejo de administración de las sociedades cotizadas no podrá delegar las facultades de decisión a que se refiere el artículo 249 bis ni específicamente a) La aprobación del plan estratégico o de negocio, los objetivos de gestión y presupuesto anuales, la política de inversiones y de financiación, la política de responsabilidad social corporativa y la política de dividendos.

Es decir, que las responsabilidades que se deriven de la falta de aprobación, supervisión o control en las materias indelegables se exigirán por las vías correspondientes directamente al Consejo. Otro paso determinante para atribuir las responsabilidades al Consejo se produce a través de Ley Orgánica 1/2015, de 30 de marzo, de modificación del Código Penal, que introduce la figura del "cumplimiento penal" en Artículo 31 bis. En concreto, en el apartado 1 de este artículo se establece la responsabilidad penal de las personas jurídicas a través de los administradores por razón de la comisión de determinados delitos (entre los que se incluyen los medioambientales -artículo 328 
del código penal-, o el tráfico de influencias -artículo 430-). Adicionalmente, en el apartado 2, se recogen las eximentes de esta responsabilidad penal de las empresas en caso de que el consejo de administración pudiera acreditar "diligencia debida".

Dado que un buen gobierno corporativo es clave para lograr la sostenibilidad corporativa, un gobierno corporativo responsable — consciente de su poder de decisión — contribuye a aumentar la transparencia y limitar los posibles riesgos de corrupción. Y esa responsabilidad le debe lleva a tomar medidas que tienen correspondencia con las metas del ODS 16, Reducir considerablemente la corrupción y el soborno en todas sus formas (16.5) y Crear a todos los niveles instituciones eficaces y transparentes que rindan cuentas (16.6). De hecho, alejarse de dichos objetivos puede impedir conseguir otros objetivos y metas como, por ejemplo, el ODS 10 y su meta 10.4, Adoptar políticas, especialmente fiscales, salariales y de protección social, y lograr progresivamente una mayor igualdad, con consecuencias en la lucha contra la pobreza y la reducción de la desigualdad.

\section{INNOVACIÓN CONTRA LA CORRUPCIÓN}

El ODS 9 plantea que la innovación y el progreso tecnológico son claves para descubrir soluciones duraderas para los desafíos económicos y medioambientales, como el aumento de la eficiencia energética y de recursos. La meta 9.5 propone Aumentar la investigación científica y mejorar la capacidad tecnológica de los sectores industriales de todos los países, en particular los países en desarrollo, entre otras cosas fomentando la innovación. Pero no solo la tecnología e innovación contribuye a la sostenibilidad ambiental; la tecnología e innovación pueden luchar contra la corrupción. El ODS 9 queda conectado con el 16: la tecnología puede reducir diversas formas de corrupción, aumentar la transparencia, mejorar la rendición de cuentas y fortalecer las instituciones. De hecho, los países más innovadores ocupan mejores posiciones en los índices de corrupción (ver Tabla 2)

Tabla 2. Los países europeos más innovadores son países menos corruptos

\begin{tabular}{|c|c|c|}
\hline PAÍS EUROPEO & INNOVACION & CORRUPCIÓN \\
\hline SUIZA & 1 & 3 \\
\hline SUECIA & 2 & 3 \\
\hline FINLANDIA & 3 & 2 \\
\hline DINAMARCA & 4 & 1 \\
\hline HOLANDA & 5 & 5 \\
\hline LUXEMBURGO & 6 & 6 \\
\hline BÉLGICA & 7 & 9 \\
\hline REINO UNIDO & 8 & 8 \\
\hline NORUEGA & 9 & 4 \\
\hline ALEMANIA & 10 & 6 \\
\hline AUSTRIA & 11 & 8 \\
\hline ISLANDIA & 12 & 7 \\
\hline
\end{tabular}

Elaboración propia a partir de los datos de Transparencia Internacional, 2019 y European Innovation Scoreboard, 2020 
Fernández Mateo, Jambrina Rodríguez

Figura 1. Innovation Index

Innovation index

Source: European Innovation Scoreboard 2020

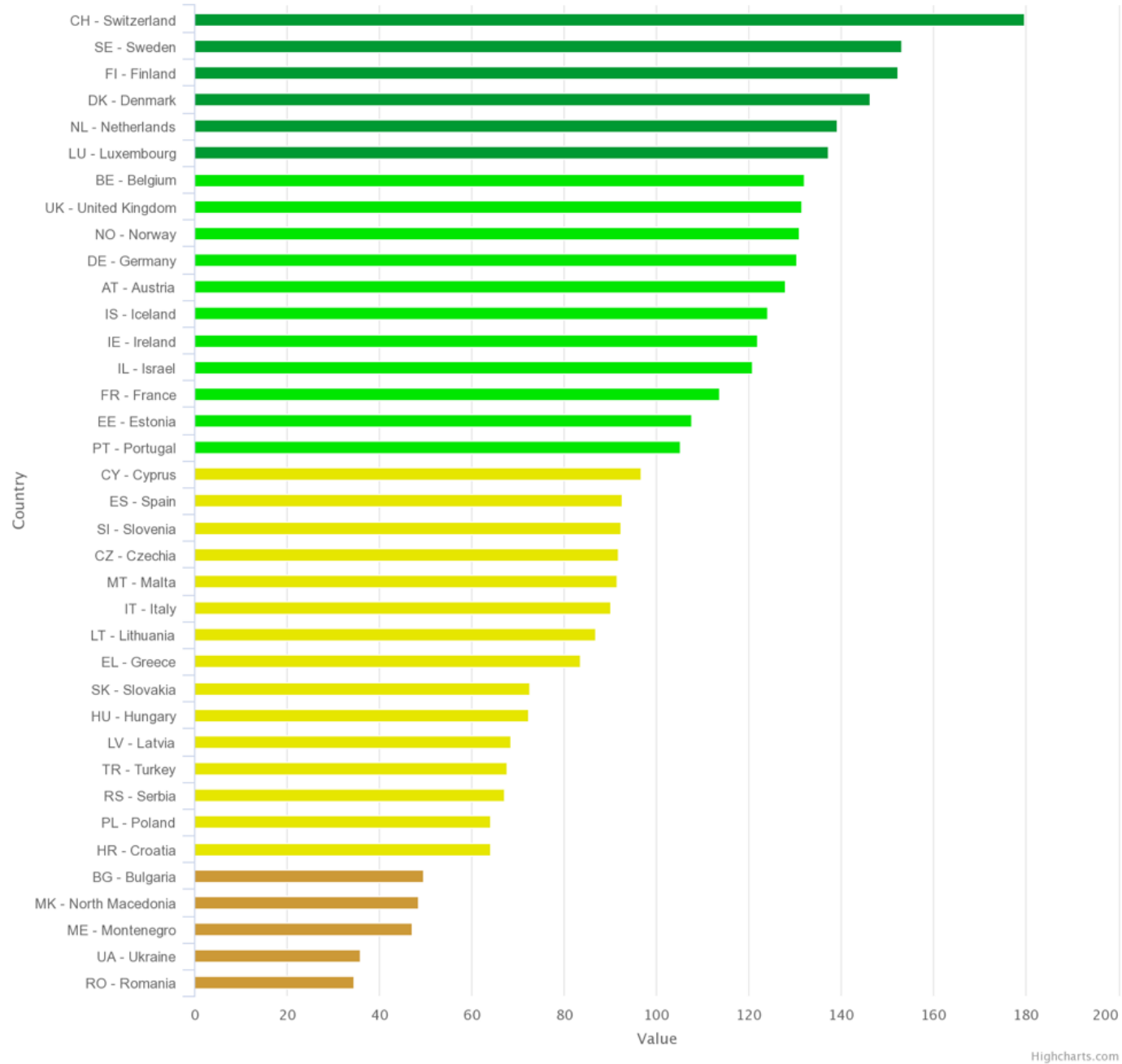

Fuente: European Innovation Scoreboard, 2020 
Figura 2. Corruption Perceptions Index

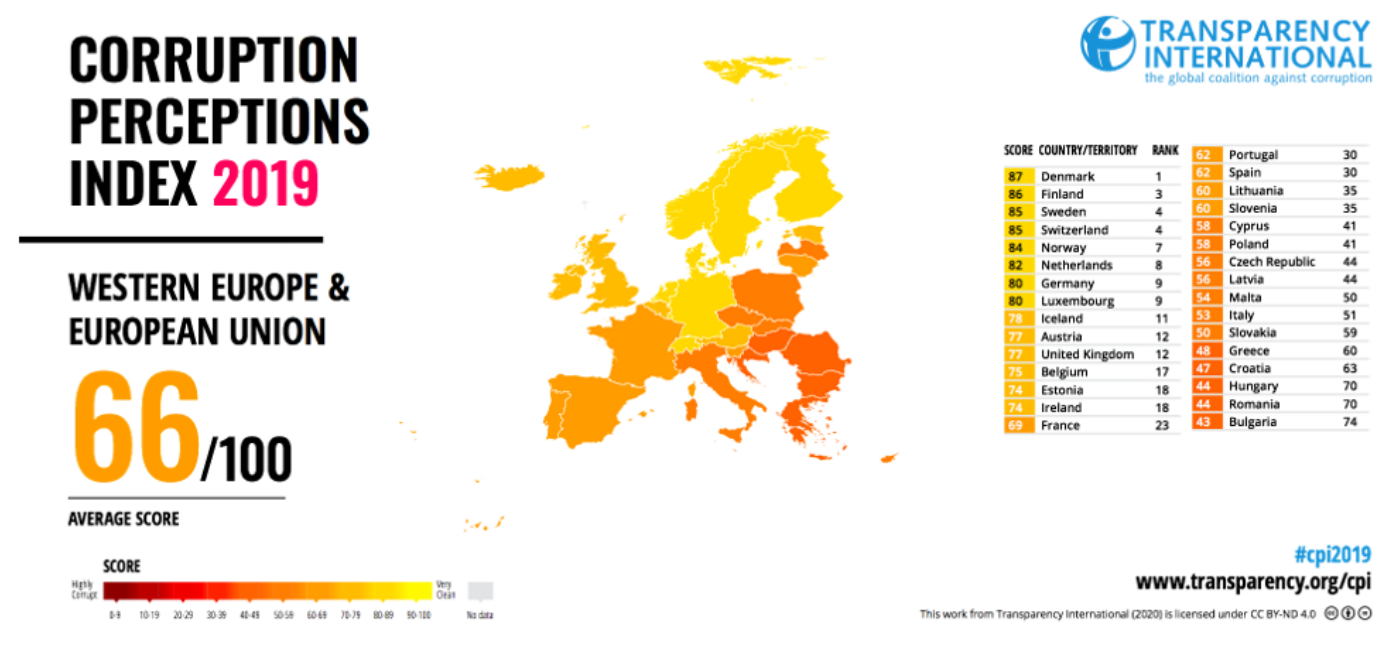

Fuente: Transparencia Internacional, 2019.

Tecnologías como la robótica, la inteligencia artificial, el blockchain, el big data, el internet de las cosas, la impresión 3D, los vehículos autónomos, la nanotecnología o la biotecnología son claves en el nuevo escenario tecnológico de la Cuarta Revolución Industrial (Lasi, Fettke, Kemper, Feld \& Hoffmann, 2014; Brynjolfsson \& McAfee, 2014; Fuchs, 2018; Cabeças, \& da Silva, 2020). En el pasado las tecnologías digitales fueron herramientas utilizadas por las organizaciones para actividades concretas o particulares. Hoy forman parte de la columna vertebral de las organizaciones y la mayoría de los procesos de la organización involucran actividades digitales. Aparecen nuevas formas de tratamiento de datos y da lugar a nuevos códigos éticos y formas de supervisión. La transformación digital y el big data permite nuevas herramientas de gestión de datos que puede prevenir el fraude en las organizaciones: por ejemplo, detectar movimientos anómalos puede permitir descubrir transacciones sospechosas. El desarrollo de software y la minería de datos posibilita cruzar datos de diversas instituciones - públicas y privadas - identificando operaciones susceptibles de fraude o irregularidades. De hecho,

The use of data analysis processes and the software dedicated to these operations provide extensive and indepth analysis of the phenomena and processes of the informal economy, fraud and corruption, as the information and communication technology becomes a sine qua non instrument of registered (formal) economy. Although on the analytical market, there is a wide spectrum of specialized tools capable to support and enhance the antifraud activity, unfortunately, the survey results indicate that managers are not taking advantage of them (Bănărescu, 2015: 1828)

¿Cómo puede contribuir la tecnología a la lucha contra la corrupción? A continuación, se enumeran aspectos esenciales posibilitados por las nuevas tecnologías:

- La automatización puede reducir las oportunidades de corrupción al detectar operaciones extrañas, anomalías o valores atípicos.

- Las innovaciones tecnológicas pueden ser utilizadas por organizaciones públicas y privadas para mejorar la eficiencia y la transparencia, comunicarse mejor con los ciudadanos y clientes, y ofrecerles más y mejor información. La transparencia puede ayudar a reducir el margen de discrecionalidad. 
- La detección preventiva a través de la vigilancia de las redes y los individuos. La innovación ofrece novedosas formas de supervisión de las actividades de las organizaciones. La alta dirección puede dotarse de mecanismos digitales para asegurar que se cumplen los objetivos corporativos a todos los niveles.

- $\quad$ La creación de canales anónimos de denuncia (Whistleblowing) permite conocer cuándo se producen casos que infringen el código ético de las compañías. Al conocer los problemas se pueden modificar las conductas, identificando errores y riesgos, y corrigiendo las situaciones.

- Los efectos disuasorios de la tecnología. La existencia de plataformas con información pública sobre casos de corrupción otorga visibilidad y disuade a los posibles infractores, aumentando la sensibilización ante el problema tanto de los miembros de las organizaciones como de los diversos stakeholders.

- La promoción de actitudes éticas mediante el compromiso público y los debates en plataformas virtuales. 
Tabla 3. La innovación tecnológica en la lucha contra la corrupción

\begin{tabular}{|c|c|c|c|}
\hline Acción & Beneficios & $\begin{array}{l}\text { Objetivo } \\
\text { principal }\end{array}$ & Plataforma \\
\hline Automatización & $\begin{array}{l}\text { Eliminar los agentes } \\
\text { humanos y, por } \\
\text { tanto, las } \\
\text { oportunidades de } \\
\text { corrupción en las } \\
\text { operaciones }\end{array}$ & $\begin{array}{l}\text { Pequeños } \\
\text { sobornos en } \\
\text { operaciones } \\
\text { cotidianas }\end{array}$ & $\begin{array}{l}\text { Cualquier } \\
\text { sistema }\end{array}$ \\
\hline Transparencia & $\begin{array}{l}\text { Visibilizar } \\
\text { actividades } \\
\text { susceptibles de } \\
\text { corrupción }\end{array}$ & $\begin{array}{l}\text { Informar a los } \\
\text { públicos }\end{array}$ & $\begin{array}{l}\text { Sitios web } \\
\text { donde se } \\
\text { publica la } \\
\text { información }\end{array}$ \\
\hline $\begin{array}{l}\text { Detección de } \\
\text { operaciones } \\
\text { fraudulentas }\end{array}$ & $\begin{array}{l}\text { Monitorear } \\
\text { movimientos } \\
\text { anómalos e } \\
\text { imprevistos }\end{array}$ & $\begin{array}{l}\text { Tanto pequeños } \\
\text { sobornos como } \\
\text { operaciones a gran } \\
\text { escala }\end{array}$ & $\begin{array}{l}\text { Herramientas } \\
\text { de análisis de } \\
\text { registros. } E \text { - } \\
\text { procurement } \\
\text { systems }\end{array}$ \\
\hline $\begin{array}{l}\text { Detección } \\
\text { preventiva }\end{array}$ & $\begin{array}{l}\text { El monitoreo y } \\
\text { detección en línea } \\
\text { de individuos } \\
\text { permite } \\
\text { identificar los } \\
\text { preparativos para } \\
\text { una acción corrupta }\end{array}$ & $\begin{array}{l}\text { Corrupción a gran } \\
\text { escala, } \\
\text { por ejemplo, en la } \\
\text { contratación } \\
\text { pública o } \\
\text { comercio } \\
\text { internacional }\end{array}$ & $\begin{array}{l}\text { Análisis de } \\
\text { redes sociales }\end{array}$ \\
\hline Disuasión & $\begin{array}{l}\text { Si el público es } \\
\text { consciente de la } \\
\text { existencia de estos } \\
\text { mecanismos, se } \\
\text { genera un efecto } \\
\text { disuasorio }\end{array}$ & $\begin{array}{l}\text { Pequeños } \\
\text { sobornos }\end{array}$ & $\begin{array}{l}\text { Cualquier } \\
\text { tecnología, } \\
\text { especialment } \\
\text { e la } \\
\text { publicación } \\
\text { en sitios web } \\
\text { y redes } \\
\text { sociales }\end{array}$ \\
\hline Informar & $\begin{array}{l}\text { La información } \\
\text { disponible permite } \\
\text { movilizar a usuarios } \\
\text { y tomar medidas } \\
\text { correctivas }\end{array}$ & $\begin{array}{l}\text { Pequeños } \\
\text { sobornos y } \\
\text { operaciones a gran } \\
\text { escala }\end{array}$ & $\begin{array}{l}\text { Sitios web, } \\
\text { redes } \\
\text { sociales, } \\
\text { prensa } \\
\text { digital, etc. }\end{array}$ \\
\hline
\end{tabular}

Elaboración propia a partir de Grönlund, 2010.

\section{CONCLUSIÓN}

La inversión en investigación, desarrollo e innovación (I+D+i) es un indicador para identificar los países que dedican parte de sus presupuestos a inversiones de alto valor añadido. La dedicación de una partida presupuestaria a la investigación y la innovación supone el desarrollo de una industria tecnológica más avanzada y competitiva que - a la luz de los datos- es directamente una política anticorrupción. 
Al mismo tiempo, el nuevo gobierno corporativo debe aplicar un concepto de sostenibilidad normativo, y aprovechar las posibilidades que ofrecen las nuevas tecnologías digitales (H2), que permiten la supervisión y control de las organizaciones, evitando los riesgos de corrupción que comprometan la sostenibilidad corporativa. Los consejos de administración, responsables de la política de RSC — facultad indelegable - pueden establecer - gracias a la innovación tecnológica - mecanismos de supervisión, evaluación y monitorización para garantizar el cumplimiento de los códigos éticos en el nuevo ecosistema digital. Las nuevas tecnologías facilitarían la implementación de esas políticas y, por tanto, asegurarían la sostenibilidad corporativa de las compañías, con efecto en las metas globales de los ODS.

El ODS 17 plantea las alianzas para lograr los objetivos deseados. Específicamente, la meta 17.17 plantea Fomentar y promover la constitución de alianzas eficaces en las esferas pública, públicoprivada y de la sociedad civil, aprovechando la experiencia y las estrategias de obtención de recursos de las alianzas. La inversión pública en $\mathrm{I}+\mathrm{D}+\mathrm{i}$ conjuntamente con la innovación privada para el logro de la sostenibilidad corporativa es una sinergia que puede impulsar las metas 17.6 mejorar la cooperación en materia de tecnología, ciencia e innovación, 17.7promover la transferencia de tecnologías y 17.8 aumentar la utilización de las tecnologías de la información y la comunicación, disminuyendo los riesgos de corrupción en organizaciones y países, aumentando la transparencia y facilitando la rendición de cuentas.

\section{REFERENCIAS BIBLIOGRÁFICAS}

Andreu Pinillos, A., Fernández Fernández, J. L., \& Fernández Mateo, J. (2018). Revisión crítica de la dimensión Gobierno Corporativo en los cuestionarios de los índices de Sostenibilidad. Revista de Comunicación, 17(2), 09-40. http://dx.doi.org/10.26441/RC17.2-2018-A1

Andreu Pinillos, A., Fernández-Fernández, J. L., \& Fernández-Mateo, J. F. (2019). Pasado, presente y futuro de los objetivos del desarrollo sostenible (ODS). La tecnología como catalizador (o inhibidor) de la Agenda 2030. Icade. Revista de la Facultad de Derecho, (108). https://doi.org/10.14422/icade.i108.y2019.001

Andreu-Pinillos, A., Fernández-Fernández, J.-L., \& Fernández-Mateo, J. (2020). Corporate governance in sustainability indexes: a Spanish case study. Revista De Comunicación, 19(2), 7-28. https://doi.org/10 .26441/RC19.2-2020-A1

Bachrach, P., \& Baratz, M. S. (1970). Power and poverty: Theory and practice. Oxford, UK: Oxford University Press; 1970.

Bănărescu, A. (2015). Detecting and Preventing Fraud with Data Analytics. Procedia Economics and Finance, (32), 1827-1836.https://doi.org/10.1016/S2212-5671(15)01485-9

Bañon Gomis, A. J., Guillén Parra, M., Hoffman, W. M., \& McNulty, R. E. (2011). Rethinking the concept of sustainability. Business and Society Review, 116(2), 171-191. https://doi.org/10.1111/j.1467-8594.20 11.00381.x

Beltratti, A. (2005). The complementarity between corporate governance and corporate social responsibility. The Geneva Papers on Risk and Insurance-Issues and Practice, 30(3), 373-386. https://doi.org/10.105 7/palgrave.gpp.2510035

Benavides Delgado, J. \& Fernández Mateo, J. (2020). Los límites de la sostenibilidad. Navarra: EUNSA

Bird, F. B. (1996). The muted conscience: Moral silence and the practice of ethics in business. Westport, CT, US: Greenwood Publishing Group.

Blair, M. M. (1995). Rethinking Assumptions Behind Corporate Governance. Challenge, 38(6), 12-17. https:/ /doi.org/10.1080/05775132.1995.11471861 
Bovens, M. (2007). Analysing and assessing accountability: A conceptual framework. European Law Journal, 13(4), 447-468. https://doi.org/10.1111/j.1468-0386.2007.00378.x

Bovens, M., Goodin, R. E., \& Schillemans, T. (Eds.). (2014). The Oxford handbook public accountability. Oxford University Press.

Bowen, H. R. (2013). Social responsibilities of the businessman. University of Iowa Press.

Brundtland (1987). Our common future. Oxford, UK: Oxford University Press.

Brynjolfsson, E. y McAfee, A. (2014). The Second Machine Age: Work, Progress, and Prosperity in a Time of Brilliant Technologies. New York: W.W. Norton \& Company.

Cabeças, A., \& da Silva, M. M. (2020). Project Management in the Fourth Industrial Revolution. TECHNO REVIEW. International Technology, Science and Society Review, 9(2), 79-96. https://doi.org/10.3746 7/gka-revtechno.v9.2804

Carroll, A. B. (1979). A three-dimensional conceptual model of corporate performance. Academy of management review, 4(4), 497-505. https://doi.org/10.5465/amr.1979.4498296

Choi, J. S., Kwak, Y. M., \& Choe, C. (2010). Corporate social responsibility and corporate financial performance: Evidence from Korea. Australian Journal of Management, 35(3), 291-311. https://doi.org/10.1177/0 312896210384681

CNMV (2015). Código de buen gobierno de las sociedades cotizadas. Comisión Nacional del Mercado de Valores. Retrieved from: https://www.cnmv.es/docportal/publicaciones/codigogov/codigo_buen_gob ierno.pdf

Dahl, R. A. (1958). A critique of the ruling elite model. The American Political Science Review, 52(2), 463-469. https://doi.org/10.2307/1952327

Dahlsrud, A. (2008). How corporate social responsibility is defined: an analysis of 37 definitions. Corporate Social Responsibility and Environmental Management, 15(1), 1-13. https://doi.org/10.1002/csr.132

Díaz-De la Cruz, C., \& Fernández-Fernández, J. L. (2016). Marco conceptual de la ética y la responsabilidad social empresarial: un enfoque antropológico y estratégico. Empresa y Humanismo, 19 (2), 69-118. $\underline{\mathrm{h}}$ ttps://doi.org/10.15581/015.XIX.2.69-118

Dyllick, T., \& Hockerts, K. (2002). Beyond the business case for corporate sustainability. Business Strategy and The Environment, 11(2), 130-141. https://doi.org/10.1002/bse.323

Eccles, R. G., Ioannou, I., \& Serafeim, G. (2014). The impact of corporate sustainability on organizational processes and performance. Management Science, 60(11), 2835-2857. https://doi.org/10.1287/mnsc .2014 .1984

Elkington J. (1997). Cannibals with Forks: the Triple Bottom Line of 21st Century Business. Capstone: Oxford.

Elkington, J. (2006). Governance for sustainability. Corporate Governance: an International Review, 14(6), 522-529. https://doi.org/10.1111/j.1467-8683.2006.00527.x

European Innovation Scoreboard (2020) Innovation Index. Retrieved from: https://interactivetool.eu/EIS /EIS_2.html\#a

Feathers, B. (2014). Bribes Without Borders: The Challenge of Fighting Corruption in the Global Context. International Law Review, 29(2), 287-292.

Fernández-Fernández, JL. (1999) Ethics and the Board of Directors in Spain: The Olivencia Code of Good Governance. Journal of Business Ethics 22, 233-247. https://doi.org/10.1023/A:1006290615353

Fernández-Fernández, J. F., \& Bajo-Sanjuán, A. (2012). La teoría del stakeholder o de los grupos de interés, pieza clave de RSE, del éxito empresarial y de la sostenibilidad. aDResearch: Revista Internacional de Investigación en Comunicación, (6), 130-143. https://doi.org/10.7263/adresic-006-02 
Fernández-Mateo, J., \& Franco-Barrera, A. J. (2020). Animal Welfare for Corporate Sustainability: The Business Benchmark on Farm Animal Welfare. Journal of Sustainability Research, 2(3). https://doi.org /10.20900/jsr20200030

Freeman, R.E. (1984). Strategic Management: a stakeholder approach. Boston, MA. Pittman.

Friedman, M. (1962). Capitalism and Freedom. University of Chicago Press.

Fuchs, C. (2018). Industry 4.0: The Digital German Ideology. TripleC: Communication, Capitalism \& Critique, 16(1), 280-289. https://doi.org/10.31269/triplec.v16i1.1010

Grönlund, Å. (2010). Using ICT to combat corruption-Tools, methods and results. In SPIDER: Increasing transparency \& fighting corruption through ICT, SPIDER

ICT4D Series no.3. Stockholm, Sweden: Stockholm University. Retrieved from: http://citeseerx.ist.psu.edu/ viewdoc/download?doi=10.1.1.465.9519\&rep=rep1\&type=pdf\#page=13

Harjoto, M. A. \& Jo, H. (2011). Corporate governance and CSR nexus. Journal of Business Ethics, 100, 45-67. https://doi.org/10.1007/s10551-011-0772-6

Hoffman, W. M. (1986). What is necessary for corporate moral excellence?. Journal of Business Ethics, 5(3), 233-242. https://doi.org/10.1007/BF00383631

Inoue, Y., \& Lee, S. (2011). Effects of different dimensions of corporate social responsibility on corporate financial performance in tourism-related industries. Tourism Management, 32(4), 790-804. https://d oi.org/10.1016/j.tourman.2010.06.019

Jo, H. \& Harjoto, M. A. (2012). The causal effect of corporate governance on corporate social responsibility. Journal of Business Ethics, 106(1), 53-72. https://doi.org/10.1007/s10551-011-1052-1

Khan, M., Serafeim, G., \& Yoon, A. (2016). Corporate sustainability: First evidence on materiality. The Accounting Review, 91(6), 1697-1724. https://doi.org/10.2308/accr-51383

Lasi, H., Fettke, P., Kemper, H. G., Feld, T. y Hoffmann, M. (2014). Industrie 4.0. Wirtschaftsinf 56, 261-264. $\underline{\mathrm{h}}$ ttps://doi.org/10.1007/s11576-014-0424-4

Lukes, S. (2005). Power: A radical view. 2nd Edition. London: Palgrave Macmillan.

Maas, K., \& Rosendaal, S. (2016). Sustainability targets in executive remuneration: Targets, time frame, country and sector specification. Business Strategy and the Environment, 25(6), 390-401. https://doi. org/10.1002/bse.1880

Marti, C. P., Rovira-Val, M. R., \& Drescher, L. G. (2015). Are firms that contribute to sustainable development better financially?. Corporate Social Responsibility and Environmental Management, 22(5), 305-319. $\underline{\mathrm{h}}$ ttps://doi.org/10.1002/csr.1347

McGrath, S. K., \& Whitty, S. J. (2018). Accountability and responsibility defined. International Journal of Managing Projects in Business, 11(3), 687-707. https://doi.org/10.1108/IJMPB-06-2017-0058

Moneva, J. M., Archel, P., \& Correa, C. (2006). GRI and the camouflaging of corporate unsustainability. Accounting Forum, 30 (2), 121-137. https://doi.org/10.1016/j.accfor.2006.02.001

Nigam, N., Benetti, C., \& Mbarek, S. (2018). Can linking executive compensation to sustainability performance lead to a sustainable business model? Evidence of implementation from enterprises around the world. Strategic Change, 27(6), 571-585. https://doi.org/10.1002/jsc.2240

O'Mara-Shimek, M., Guillén, M., \& Bañón Gomis, A. J. (2015). Approaching virtuousness through organizational ethical quality: toward a moral corporate social responsibility. Business Ethics: A European Review, 24, S144-S155. https://doi.org/10.1111/beer.12102

Polsby, N. W. (1963). Community Power and Political Theory. New Haven, CT: Yale University Press.

Shleifer, A., \& Vishny, R. W. (1997). A survey of corporate governance. The Journal of Finance, 52(2), 737-783. https://doi.org/10.1111/j.1540-6261.1997.tb04820.x 
Stanwick, P. A., \& Stanwick, S. D. (1998). The relationship between corporate social performance, and organizational size, financial performance, and environmental performance: An empirical examination. Journal of Business Ethics, 17(2), 195-204. https://doi.org/10.1023/A:1005784421547

Tourigny, L., Dougan, W. L., Washbush, J., \& Clements, C. (2003). Explaining executive integrity: governance, charisma, personality and agency. Management Decision. 41(10), 1035-1049. https://doi.org/10.1108 /00251740310509562

Transparency International (2019) Corruption Pereception Index. Retrieved from https://www.transparen cy.org/en/cpi/2019/index/nz

Trapnell, S., Jenkins, M., \& Chene, M. (2017). Monitoring corruption and anti-corruption in the Sustainable Development Goals. Berlin: Transparency International. Retrieved from: https://www.transparency.or g/en/publications/monitoring-corruption-and-anti-corruption-in-the-sustainable-development-go\#

Useem, M., \& Zelleke, A. (2006). Oversight and delegation in corporate governance: Deciding what the board should decide. Corporate Governance: An International Review, 14(1), 2-12. https://doi.org/10.1111/ j.1467-8683.2006.00479.x

Viederman, S. (1994). The economics of sustainability: challenges. Paper presented at the Workshop, The Economics of Sustainability, Fundação Joaquim Nabuco, Recife, Brazil.

Waddock, S. A., \& Graves, S. B. (1997). The corporate social performance-financial performance link. Strategic Management Journal, 18(4), 303-319. https://doi.org/10.1002/(SICI)1097-0266(199704)1 8:4303::AID-SMJ8693.0.CO;2-G

NOTAS

* Joaquín Fernández Mateo es Doctor por la Universidad Complutense de Madrid (2014). Profesor del Departamento de Ciencias de la Educación, Lenguaje, Cultura y Artes, Ciencias Histórico-Jurídicas y Humanísticas y Lenguas Modernas de la Universidad Rey Juan Carlos de Madrid. Miembro del Grupo de investigación La persona en la Filosofía española contemporánea: Vida humana y Bioderecho de la Universidad Rey Juan Carlos. Es autor, junto a Juan Benavides Delgado, del libro Los límites de la sostenibilidad, editado por EUNSA.

** Julia Jambrina Rodríguez es doctora en Las Actividades Físicas y Artísticas (Educación Musical) por la Universidad de Extremadura (UEX), Máster Universitario en Formación de Profesorado de Educación Secundaria Obligatoria y Bachillerato por la Universidad Autónoma de Madrid (UAM), Licenciada en Derecho por la Universidad Autónoma de Madrid (UAM) y abogada colegiada ejerciente por el Ilustre Colegio de Abogados de Madrid (ICAM). 